\title{
IgA, cytokines and growth factors profile in term, preterm and high preterm human colostrum
}

\author{
C. Ramírez-Santana ${ }^{1,2}$, R. Casillas ${ }^{1,2}$, C. Moltó-Puigmartí ${ }^{2,3}$, X. Carbonell-Estrany ${ }^{4}$, M. Castell ${ }^{1}$, \\ M. C. López-Sabater ${ }^{2,3}$, C. Castellote ${ }^{1,2}$ and A. Franch ${ }^{1,2}$ \\ ${ }^{1}$ Department of Physiology, Faculty of Pharmacy, University of Barcelona, Spain, ${ }^{2}$ CIBER in Epidemiology and Public \\ Health (CIBERESP), Spain, ${ }^{3}$ Department of Nutrition and Food Science, Faculty of Pharmacy, University of Barcelona, \\ Spain and ${ }^{4}$ Neonatology Service, Clínic-Maternitat Hospital of Barcelona, Spain
}

Human milk provides infants with nutrients suited to meet developmental needs. Less well understood are the immunological and defensive functions of milk. Milk contains many immunoactive molecules including antibodies, cytokines, chemokines and growth factors, which have yet unexplained roles in infant physiology. The present study was designed to determine IgA, EGF, TGF- $\beta 1$, TGF- $\beta 2$, IL-6, IL-8, IL-10, IL-13, TNF- $\alpha$ and TNF-RI colostrum contents, in order to compare differences among mothers' milk of term, preterm and high preterm infants.

Colostrum samples were collected during hospitalisation by an electric breast pump, and obtained 2-3 days postpartum from 22 breastfeeding mothers who delivered at term (group T), 10 mothers delivering between 30 and 37 weeks of gestation (group PT) and 10 mothers delivering less than 30 weeks of gestation (group HPT). Milk whey was obtained by milk centrifugation at $800 \mathrm{~g}, 10 \mathrm{~min} 4{ }^{\circ} \mathrm{C}$. IgA, EGF, TGF- $\beta 1$ and TGF- $\beta 2$ were determined by ELISA, and IL-6, IL-8, IL-10, IL-13, TNF- $\alpha$ and TNF-RI were simultaneously quantified by $\mathrm{BD}^{\mathrm{TM}}$ Cytometric Bead Array Human Soluble Flex Set Assay (Becton Dickinson, San Diego, CA, USA). Differences between groups were analysed by the Mann-Whitney $U$-test. Significant differences were accepted at $P<0.05$.

The PT group showed the highest variability in all the studied immunoactive factors, probably due to the interval of gestation period lasting considered ( 7 weeks). This group showed a significant increase of TGF- $\beta 1$ and TGF- $\beta 2$ colostrum content with respect to T group $(P<0.05)$. HPT group in relation to PT group presented reduced contents of IgA, TGF- $\beta 2$, IL-6, IL-8, IL-10 and TNF- $\alpha(P<0.05)$. No significant changes were observed for EGF and TNF-RI among groups. IL-13 was undetected in 59\%, 70\% and $90 \%$ of T, PT and HPT colostrum whey, respectively (Table). The low content of IgA and some cytokines in colostrum for feeding high preterm infants could contribute to the tendency of developing necrotizing enterocolitis of these newborns.

\begin{tabular}{|c|c|c|c|c|c|c|}
\hline \multirow[b]{3}{*}{ Immunoactive factor } & \multicolumn{6}{|c|}{ Group } \\
\hline & \multicolumn{2}{|c|}{$\mathrm{T}$} & \multicolumn{2}{|c|}{ PT } & \multicolumn{2}{|c|}{ HPT } \\
\hline & Mean & $\mathrm{SE}$ & Mean & $\mathrm{SE}$ & Mean & $\mathrm{SE}$ \\
\hline $\mathrm{IgA}(\mathrm{mg} / \mathrm{ml})$ & 6.39 & 1.12 & 9.09 & 2.32 & $2.53 * \phi$ & 0.97 \\
\hline EGF (ng/ml) & 75.59 & 11.69 & 142.50 & 36.76 & 95.60 & 17.03 \\
\hline TGF- $\beta 1$ (pg/ml) & 598.19 & 114.05 & $2809.9 *$ & 1471.81 & 692.28 & 130.81 \\
\hline TGF- $\beta 2$ (ng/ml) & 3.76 & 0.79 & $10.99 *$ & 3.69 & $2.94^{\phi}$ & 0.43 \\
\hline IL-6 (pg/ml) & 69.44 & 18.08 & 125.99 & 32.21 & $26.72^{\phi}$ & 6.03 \\
\hline IL-8 (ng/ml) & 3.77 & 1.34 & 5.24 & 1.63 & $0.62 * \phi$ & 0.27 \\
\hline IL-10 (pg/ml) & 5.92 & 1.60 & 8.80 & 2.45 & $2.00^{* \phi}$ & 0.75 \\
\hline IL-13 (pg/ml) & 6.69 & 1.71 & 8.25 & 3.92 & $1.28^{* \phi}$ & 0.68 \\
\hline TNF- $\alpha(\mathrm{pg} / \mathrm{ml})$ & 11.45 & 1.81 & 18.25 & 4.86 & $4.18^{* \phi}$ & 0.92 \\
\hline TNF-RI (ng/ml) & 1.19 & 0.19 & 2.38 & 0.84 & 1.09 & 0.11 \\
\hline
\end{tabular}

T, term group; PT, preterm group; HPT, high preterm group. $* P<0.05 v$. T group, $\phi P<0.05 v$. PT. 